\title{
VARIÁVEIS DE SAÚDE FÍSICA EM CRIANÇAS ESCOLARES COM BAIXO NÍVEL SOCIOECONÔMICO NA AMAZÔNIA-BRASILEIRA
}

\author{
Gabriel dos Santos Gonçalves ${ }^{1}$, Pedro Lucas da Costa Dias ${ }^{1}$, Roseane Monteiro - \\ Santos ${ }^{1}$, Olivar de Souza Martins ${ }^{2}$, Claudio Joaquim Borba - Pinheiro ${ }^{1,2 *}$
}

\begin{abstract}
RESUMO
GONÇALVES, G.S.; DIAS, P.L.C.; MONTREIRO-SANTOS, R.; MARTINS, O.S.; BORBAPINHEIRO, C.J. Coordenação motora grossa e estado nutricional de escolares com baixo nível socioesconômico na Amazônia brasileria . Perspectivas Online: Biológicas \& Saúde, v. 11, n. 40 , p. $39-53,2021$.

Objetivo: avaliar a coordenação motora grossa, o nível socioeconômico e estado nutricional de meninos e meninas de 8 a 10 anos de uma escola pública na periferia do município de Tucuruí-PA. Materiais e Métodos: participaram deste estudo 100 alunos do $3^{\circ}, 4^{\circ}$ e $5^{\circ}$ ano do ensino fundamental I. Para a avaliação socioeconômica foi utilizado o Critério de Classificação Econômica Brasil ABEP através de questionário. A classificação utilizada para o estado nutricional foi a recomendada pelo SISVAN do Índice de Massa Corporal (IMC) proposta pela Organização Mundial da Saúde para crianças a partir dos cinco anos. E para a avaliação da coordenação motora grossa, foi utilizado o protocolo

Resultados: o nível de coordenação motora no geral foi muito baixo, mostrando que apenas $22 \%$ ficaram dentro do normal; na força e agilidade apresentaram diferenças $(\mathrm{p}<0,05)$ favoráveis ao sexo masculino, respectivamente $(81$ e 92,3$)$ e o feminino $(71,8$ e 83,6$)$. No estado nutricional, a maioria (93\%) apresentou estado de normalidade. O nível socioeconômico indicou que as famílias dessas crianças são de baixo poder aquisitivo, tendo prevalência à classe C2 com $41 \%$. Conclusão: inferiu-se que o nível socioeconômico pode ser a causa do baixo nível de coordenação motora grossa de escolares da rede pública do ensino fundamental I de 8-10 anos do município de Tucuruí-PA.
\end{abstract} Korperkoodinationtest fur Kinder (KTK).

Palavras-chave: desempenho psicomotor, nível socioeconômico, estado nutricional

\footnotetext{
${ }^{1}$ Universidade do Estado do Pará (UEPA) campus XIII Tucuruí - PA, Brasil.J, CEP: 28035-310, Brasil;

${ }^{2}$ Instituto Federal de Educação, Ciência e Tecnologia do Pará (IFPA) Campus de Tucuruí-PA, Brasil

Autor correspondente:

Rua 8 q-11 nº $4^{\text {a }}$, Bairro:Park dos Buritis 1. CEP: 68459876.

(*) e-mail: claudioborba18@gmail.com

Data de recebimento: 26/10/2021.

Aceito para publicação: 06/11/2021.

Data de Publicação 21/12/2021
}

Persp. Online: biol \& saúde, Campos dos Goytacazes, 40 (11) 39-53, 2021

ojs3.perspectivasonline.com.br 
Revista Perspectivas Online: Biológicas \& Saúde December/2021, v. 11, n. 40 , p. $39-53$ ISSN: 2236-8868 (Online)

DOI: $10.25242 / 8868114020212470$

\title{
PHYSICAL HEALTH VARIABLES OF SCHOOLCHILDREN WITH LOW SOCIO- ECONOMIC LEVEL IN BRAZILIAN AMAZON
}

Gabriel dos Santos Gonçalves ${ }^{1}$, Pedro Lucas da Costa Dias ${ }^{1}$, Roseane Monteiro - Santos ${ }^{1}$, Olivar de Souza Martins ${ }^{2}$, Claudio Joaquim Borba - Pinheiro ${ }^{1,2 *}$

\section{RESUMO}

GONÇALVES, G.S.; DIAS, P.L.C.; MONTREIRO-SANTOS, R.; MARTINS, O.S.; BORBAPINHEIRO, C.J. Physical health variables of schoolchildren with low socio-economic level in brazilian amazon. Perspectivas Online: Biológicas \& Saúde, v. 11, n. 40 , p. 39 - 53 , 2021.

\begin{abstract}
Objective: to evaluate the gross motor coordination, socioeconomic and nutritional status of male and female aged 8 to 10 years from a public school of the city of Tucuruí-PA. Materials and Methods: 100 students from the 3rd, 4th and 5th grades of elementary school participated in this study. For the socioeconomic evaluation, the ABEP Economic Classification Criterion was used. The classification used for nutritional status was that recommended by SISVAN of the Body Mass Index (BMI) proposed by the World Health Organization for children from five years. And, for the evaluation of gross motor coordination, the

protocol was used. Results: the level of motor coordination in general was very low, showing that only $22 \%$ were within normal; presented favorable differences $(\mathrm{p}<0.05)$ for male (81 and 92.3) and female (71.8 and 83.6) respectively, for strength and agility. In the nutritional state, the majority $(93 \%)$ presented normal state. The socioeconomic level indicated that the families of these children are of low purchasing power, having a prevalence of class C2 with $41 \%$. Conclusion: it was inferred that the socioeconomic level may be the cause of the low level of gross motor coordination of 8-10 years old schoolchildren in the city of Tucuruí-PA.
\end{abstract} Korperkoodinationtest fur Kinder (KTK)

Keywords: psychomotor performance; socioeconomic level; nutritional status.

${ }^{1}$ University of State of Pará (UEPA) campus XIII Tucuruí - PA, Brazil. Postal Code 28035-310;

${ }^{2}$ IFederal Institute of Education, Science and Technology of (IFPA) Tucuruí-PA, Brazil

Autor correspondente: Street 8 q-11 District:Park dos Buritis 1 . Postal Code: 68459 876. $\quad$ (*) e-mail: claudioborba18@gmail.com

Receipt: 26/10/2021

Accepted for publication: 06/11/2021.

Date of Publicação 21/12/2021 . 


\section{INTRODUÇÃ̃O}

A infância é uma fase da vida marcada por rápidas alterações no desenvolvimento e crescimento. Este período proporciona, nas crianças, o aperfeiçoamento social, cognitivo e motor, de modo que o meio em que estão inseridas influencia na prática das áreas do movimento e aprendizagem, mantendo assim os hábitos adquiridos para à vida adulta (GALLAHUE, OZMUN; GOODWAY, 2013).

Neste sentindo, os aspectos socioeconômicos e ambientais são elementos importantes para o desenvolvimento infantil, podendo influenciar negativamente com relação ao crescimento e desenvolvimento, se estes não suprem as necessidades individuais (BRANCO, GUILHERM; CALOMENI, 2018; ESTEVÃO; DA LUZ, 2015; SOUZA et al., 2015). Sendo assim, o poder aquisitivo familiar está associado com a motricidade fina e ampla, desde modo, entende-se que é essencial estudos a respeito das consequências do nível socioeconômico e da coordenação motora (GIORDANI, ALMEIDA; PACHECO, 2013; SOUZA et al., 2015). É importante destacar, que existe uma grande desigualdade econômica na sociedade brasileira (GIORDANI et al., 2013), e de acordo com o Instituto Brasileiro de Geografia e Estatística (IBGE, 2013), cerca de 20 milhões de indivíduos melhoraram de classe social, fato que possivelmente pode está relacionado às políticas públicas do Brasil.

Além disso, o estado nutricional é outra variável que pode ter influência no crescimento e desenvolvimento infantil, pois é a condição que o organismo assume em decorrência da sua nutrição e gasto metabólico, representando a interação de elementos somáticos e funcionais responsáveis pela absorção de nutrientes e adequação das necessidades fisiológicas do indivíduo (LIMA, 2014; ZANATO, DE OLIVEIRA; CALÁBRIA, 2018; SANTOS, 2014). Sendo assim, a avaliação do estado nutricional é fundamental para estudos que envolvem crianças, a fim de observar se o crescimento está acompanhando o padrão esperado ou se afastando por doença e/ou por condições sociais desfavoráveis. Além do mais, uma condição nutricional inapropriada, se expõe a complicações de desnutrição ou abundância de gordura, que aliado a distúrbios no andamento da coordenação motora poder causar ou intensificar o denominado Transtorno de Desenvolvimento da Coordenação (TDC) (EDITION et al., 2013; RIBEIRO et al., 2012; SILVA; BELTRAME, 2013).

Outra variável que influencia o desenvolvimento infantil é a coordenação motora grossa que se caracteriza como um sistema linear e consecutivo vinculado à idade cronológica, na qual, já se sabe as inúmeras capacidades e habilidades motoras que devem estar aguçadas, sendo elas básicas e complexas, com a finalidade de gerar ações de locomoção com precisão, força, agilidade, noção espaço-temporal e equilíbrio (RIBEIRO et al., 2012; SILVA, J.; BELTRAME, 2013). Esses aspectos se tornam ainda mais importantes quando, de acordo com as considerações de Ribeiro et al. (2012) a capacidade reduzida de equilíbrio postural, as deficiências na fluidez do movimento, a falta de precisão da direção, de força e de agilidade podem estar associadas, dentre outros fatores, a uma coordenação motora grossa diminuída.

Com isso, observa-se uma possível interação entre as variantes: nível socioeconômico, estado nutricional e coordenação motora grossa, que podem provocar sérios danos ao crescimento e desenvolvimento de crianças na fase escolar (CONSTANTINO-COLEDAM et al., 2013; GORE et al., 2012) com destaque ao TDC (RIBEIRO et al., 2012). Neste cenário, o aspecto socioeconômico, a cerca de grau de escolaridade e poder financeiro, vincula-se ao desenvolvimento motor, especialmente quando agregado a um ambiente sortido de brinquedos e de instrumentos de motricidade na sociedade brasileira.

Persp. Online: biol \& saúde, Campos dos Goytacazes, 40 (11) 39-53, 2021

ojs3.perspectivasonline.com.br 
Diante disso, definiu-se o seguinte problema da pesquisa: qual será o nível socioeconômico, grau de coordenação motora grossa e estado nutricional de crianças de 8-10 anos de uma escola pública em um bairro da periferia do município de Tucuruí-PA?

Com isso, o objetivo deste estudo foi avaliar a coordenação motora grossa, o nível socioeconômico e estado nutricional de alunos de ambos os sexos de 8 a 10 anos de uma escola pública no município de Tucuruí-PA.

\section{METODOLOGIA}

\subsection{Tipo de Estudo}

Este estudo caracteriza-se como descritivo de caráter transversal. Para Gusmão (2011) a pesquisa também é caracterizada como um estudo de hipótese de associação com Interferência, uma vez que as variáveis investigadas envolvem causa-efeito em diferentes ambientes.

\subsection{Participantes}

O município estudado, conta com 59 escolas públicas do ensino fundamental, deste número, sorteou-se para a coleta de dados uma escola de zona periférica. O público alvo foram alunos do $3^{\circ}, 4^{\circ}$ e $5^{\circ}$ ano do ensino fundamental de uma escola pública da periferia de TucuruíPA. A coleta de dados ocorreu na própria escola, sendo todos os procedimentos planejados e acompanhados por pesquisadores treinados (alunos e professor) do curso de Educação Física da Universidade do Estado do Pará (UEPA).

\subsection{Critério para seleção da amostra}

Os critérios de inclusão foram: alunos regularmente matriculados na escola onde foi realizado o estudo, que tivessem entre 8 e 10 anos, de ambos os gêneros, quisessem participar da pesquisa e fossem autorizados pelos pais. Já os critérios de exclusão foram: alunos com baixa frequência escolar, alunos com algum tipo de limitação física ou mental que dificultasse ou impossibilitasse a participação das atividades propostas e aqueles com algum tipo de dispensa médica. Após os procedimentos de seleção, a amostra foi constituída de 100 alunos entre 8 e 10 anos de idade de ambos os sexos biológicos (46 masculino e 54 feminino).

\section{4 Ética da Pesquisa}

O estudo foi aprovado pelo comitê de ética do Instituto de Ciência da Saúde da universidade Federal do Pará (UFPA) $n^{\circ}$ 358.791. Todos os responsáveis pelos escolares participantes desta pesquisa assinando um termo de consentimento livre e esclarecido em conformidade com a Resolução $n^{\circ}$ 466/12 que trata da pesquisa com seres humanos (BRASIL, 2012).

\subsection{Protocolos de Avaliação}

\subsubsection{Avaliação socioeconômica}

Para a avaliação socioeconômica foi utilizado o Critério de Classificação Econômica Brasil da Associação Brasileira de Empresas de Pesquisa (ABEP, 2015), que estima o poder de compra das famílias, classificando-as em classes socioeconômicas, onde estabelece a classe

Persp. Online: biol \& saúde, Campos dos Goytacazes, 40 (11) 39-53, 2021

ojs3.perspectivasonline.com.br 
"DE" como a mais baixa e a classe "A" como a mais alta. Para tanto, foi feito o uso do sistema de pontuações que relaciona: o grau de instrução do chefe de família, quantidade de banheiros, posse de bens duráveis, empregada doméstica e acesso a serviços públicos (água encanada e rua pavimentada). Além de proporcionar uma previsão de renda familiar estimada. Os pontos encontrados para cada item são somados para classificação da classe econômica de cada participante.

\subsubsection{Avaliação do estado nutricional}

A avaliação do estado nutricional foi realizada através da verificação da massa corporal total, estatura e cálculo de IMC (Massa/Estatura $(m)^{2}$ ) dos escolares determinados em balança antropométrica G-TECH, capacidade de $150 \mathrm{~kg}$ e intervalos de $100 \mathrm{~g}$, com o indivíduo descalço, usando roupas leves, de pé, com os calcanhares juntos e a cabeça posicionada no plano horizontal. A estatura foi verificada utilizando uma trena da marca Tramontina de $5 \mathrm{~m}$, escalonada em $\mathrm{cm}$ e mm, sendo o marco $(0)$ zero na inserção do solo à parede, assim com o sujeito de pé e calcanhares unidos e junto à parede, com tronco e cabeça eretos, verificou-se a estatura. Os índices antropométricos mais amplamente usados, recomendados pela OMS (YAN-PING et al., 2009), adotados pelo Ministério da Saúde para a avaliação do estado nutricional de crianças e utilizados neste trabalho foram:

Índice de Massa Corporal (IMC) para idade: expressa a relação entre o peso da criança e o quadrado da estatura. É utilizado para identificar o excesso de peso entre crianças e tem a vantagem de ser um índice que será utilizado em outras fases do curso da vida. Estatura para idade (E/I): Expressa o crescimento linear da criança. É o índice que melhor indica o efeito cumulativo de situações adversas sobre o crescimento da criança. É considerado o indicador mais sensível para aferir a qualidade de vida de uma população. Trata-se de um índice incluído recentemente na Caderneta de Saúde da Criança.

A classificação utilizada para o estado nutricional neste estudo é a recomendada pelo SISVAN do Índice de Massa Corporal - IMC proposta pela Organização Mundial da Saúde para crianças a partir dos cinco anos (YAN-PING et al., 2009). As curvas de avaliação do crescimento para crianças dos cinco aos 19 anos são as recomendadas pela OMS (YAN-PING et al., 2009).

\subsubsection{Avaliação da coordenação motora grossa pela bateria de teste KTK (Körperkoordinationtest fürKinder)}

Para a avaliação da Coordenação Motora (CM) foi utilizada a bateria de teste KTK. Segundo Gorla et al. (2003), o KTK baseia-se em quatro atividades de mobilidade, como a Trave de Equilíbrio (TE), com o propósito de firmeza do equilíbrio em marcha para trás sobre três traves de larguras diferentes. O Salto Monopedal (SM), com o propósito de força e coordenação dos membros inferiores. O Salto Lateral (SL), com o propósito de agilidade em saltos alternados, e por fim a Transposição Lateral (TL), com propósito de lateralidade e estruturação do espaço físico temporal. O (TE) foi composto por três traves, a primeira de $6 \mathrm{~cm}$, a segunda $4,5 \mathrm{~cm}$ e última $3 \mathrm{~cm}$. Para o $(\mathrm{SM})$ utilizou-se espumas com as dimensões $50 \mathrm{~cm} \mathrm{x}$ $20 \mathrm{~cm}$ x $5 \mathrm{~cm}$. No (SL), foi delimitado no chão com uma fita um retângulo com dimensões $100 \mathrm{~cm}$ x $60 \mathrm{~cm}$ divido ao meio do lado mais comprido por um sarrafo de $60 \mathrm{~cm}$ x $4 \mathrm{~cm}$ x $2 \mathrm{~cm}$. E na (TL) foi utilizado duas placas de madeira com $25 \mathrm{~cm}$ x $25 \mathrm{~cm}$ x $1,5 \mathrm{~cm}$ de tamanho em que nas esquinas se encontram parafusadas quatro pés com $3,5 \mathrm{~cm}$ de altura.

Persp. Online: biol \& saúde, Campos dos Goytacazes, 40 (11) 39-53, 2021

ojs3.perspectivasonline.com.br 
A análise dos resultados da bateria KTK foi realizada por meio da soma dos pontos atingidos em cada uma das tentativas de cada prova e, através da relação destes com a idade dos escolares, obteve-se um quociente motor $(\mathrm{QM})$, derivado de uma tabela de referência para cada uma das quatro tarefas. A soma dos quatro QM (QM de cada tarefa) equivalerá a um escore que varia de 42 a 148 pontos, relacionados percentualmente.

\subsection{Análise Estatística}

As análises foram realizadas pelo software BioEstat $5.3^{\circledR}$. O nível de significância estatística foi de $p<0,05$. Inicialmente foi realizada uma descrição das variáveis da amostra dos grupos estudados com valores de média, desvio padrão, números mínimo e máximo. Posteriormente foi realizada uma análise de normalidade usando os testes de Shapiro-Wilk e Komoslgorov Smirnov e de acordo o resultado utilizou-se o Teste t ou Mann Whitney para amostras independentes. Foi usado, ainda, para os dados de classificação da coordenação motora grossa, avaliação socioeconômica e estado nutricional, uma estatística não paramétrica pelo teste binomial para duas proporções.

\section{RESULTADOS}

A Tabela 1 apresenta os dados descritivos com média, desvio padrão, números máximo e mínimo. Além disso, foi realizada uma análise de amostras independentes que não constatou diferenças significativas $(\mathrm{p}<0,05)$ para idade, massa, estatura, IMC com classificação de eutrofia e classe social que teve classificação C2 para ambos os sexos biológicos. Porém, para o somatório do protocolo de coordenação motora grossa (KTK) houve uma diferença estatística significativa favorável ao grupo do sexo masculino, em que ambos os grupos são classificados em perturbação motora.

Tabela 1. Dados descritivos com comparação de amostras independentes

\begin{tabular}{|c|c|c|c|c|c|c|c|c|c|}
\hline \multirow[b]{2}{*}{ Variáveis } & \multicolumn{4}{|c|}{ Masculino, 46} & \multicolumn{4}{|c|}{ Feminino, 54} & \multirow[b]{2}{*}{$\begin{array}{c}p- \\
\text { valor }\end{array}$} \\
\hline & Média & DP & Máx. & Mín. & Média & DP & Máx. & Mín. & \\
\hline Idade (anos) & 9,6 & 0,81 & 10,9 & 8,1 & 9,5 & 0,76 & 10,9 & 8,2 & 0,406 \\
\hline Massa (kg) & 33,6 & 9,9 & 73,8 & 22,0 & 31,3 & 7,3 & 67,0 & 21,7 & 0,197 \\
\hline Estatura (m) & 1,39 & 0,09 & 1,60 & 1,20 & 1,36 & 0,07 & 1,52 & 1,19 & 0,106 \\
\hline $\operatorname{IMC}\left(\mathrm{kg} / \mathrm{m}^{2}\right)$ & 17,07 & 3,1 & 29,5 & 12,2 & 16,7 & 2,8 & 29,7 & 12,5 & 0,580 \\
\hline Classe Social (escore) & 21,04 & 6,5 & 38,0 & 10,0 & 21,9 & 6,7 & 43,0 & 11,0 & 0,485 \\
\hline Somatório KTK (Pontos) & 346,9 & 35,6 & 424,0 & 279,0 & 324,7 & 40,5 & 416,0 & 224,0 & 0,004 \\
\hline
\end{tabular}

Siglas: IMC- índice de massa corporal; KTK - körperkoordination test für kinder; Máx. - máximo; Mín.mínimo.

A Tabela 2 apresenta os resultados para as classificações de desempenho da coordenação motora grossa e do estado nutricional. Além disso, também foi apresentado uma comparação entre os dados dos grupos masculino e feminino. Para a coordenação verificou-se que a maior parte dos alunos do sexo masculino apresentou escores classificados como perturbação (61\%), insuficiência (13\%) e 26\% mostrou normalidade na coordenação. Resultados semelhantes foram encontrados para o sexo feminino: perturbação (45\%), insuficiência (33\%) e a minoria $22 \%$ mostrou normalidade na coordenação. Cabe destacar, que

Persp. Online: biol \& saúde, Campos dos Goytacazes, 40 (11) 39-53, 2021

ojs3.perspectivasonline.com.br 
na classificação de insuficiente houve uma diferença estatística $(\mathrm{p}<0,05)$ favorável ao sexo feminino.

Os resultados para estado nutricional também estão apresentados na Tabela 2, é possível verificar que a maioria dos escolares de ambos os grupos apresentaram IMC para Idade dentro da normalidade (Eutrofismo). Porém, é importante destacar que o sexo masculino apresentou obesidade com diferença $(\mathrm{p}<0,05)$ comparado ao sexo feminino, o que não ocorreu com as outras classificações.

Tabela 2. Apresenta os resultados para Coordenação Motora Grossa, Estado Nutricional e Nível socioeconômico

\begin{tabular}{|c|c|c|c|c|c|c|c|}
\hline \multirow[b]{2}{*}{ Variáveis } & \multicolumn{2}{|c|}{ Masculino, $n=46$} & \multicolumn{2}{|c|}{ Feminino, $n=54$} & \multicolumn{2}{|c|}{ Geral, $\mathbf{n}=\mathbf{1 0 0}$} & \multirow[b]{2}{*}{ p-valor } \\
\hline & Frequência & $\%$ & Frequência & $\%$ & Frequência & $\%$ & \\
\hline \multicolumn{8}{|c|}{ Coordenação Motora Grossa (KTK) } \\
\hline Muito boa & 0 & 0 & 0 & $0 \%$ & 0 & $0 \%$ & $* * *$ \\
\hline Boa & 0 & 0 & 0 & $0 \%$ & 0 & $0 \%$ & $* * *$ \\
\hline Normal & 12 & $26 \%$ & 12 & $22 \%$ & 24 & $24 \%$ & 0,652 \\
\hline Perturbação & 28 & $61 \%$ & 24 & $45 \%$ & 52 & $52 \%$ & 0,101 \\
\hline Insuficiente & 6 & $13 \%$ & 18 & $33 \%$ & 24 & $24 \%$ & $\mathbf{0 , 0 1 7}$ \\
\hline Total & 46 & $100 \%$ & 54 & $100 \%$ & 100 & $100 \%$ & \\
\hline \multicolumn{8}{|c|}{ Estado Nutricional (SISVAN) } \\
\hline \multicolumn{8}{|c|}{ IMC/Idade } \\
\hline Eutrófico & 33 & $72 \%$ & 40 & $74 \%$ & 73 & $73 \%$ & 0,793 \\
\hline Sobrepeso & 5 & $11 \%$ & 9 & $17 \%$ & 14 & $14 \%$ & 0,405 \\
\hline Obesidade & 6 & $13 \%$ & 0 & $0 \%$ & 6 & $6 \%$ & $\mathbf{0 , 0 2 8}$ \\
\hline $\begin{array}{l}\text { Obesidade } \\
\text { Grave }\end{array}$ & 1 & $2 \%$ & 2 & $4 \%$ & 3 & $3 \%$ & 0,654 \\
\hline Magreza & 0 & $0 \%$ & 3 & $5 \%$ & 3 & $3 \%$ & 0,389 \\
\hline $\begin{array}{l}\text { Magreza } \\
\text { Acentuada }\end{array}$ & 1 & $2 \%$ & 0 & $0 \%$ & 1 & $1 \%$ & 0,908 \\
\hline Total & 46 & $100 \%$ & 54 & $100 \%$ & 100 & $100 \%$ & - \\
\hline \multicolumn{8}{|c|}{ Estatura/Idade } \\
\hline Adequada & 44 & $96 \%$ & 50 & $93 \%$ & 94 & $94 \%$ & 0,766 \\
\hline Muito baixa & 0 & $0 \%$ & 0 & $0 \%$ & 0 & $0 \%$ & $* * *$ \\
\hline Baixa & 2 & $4 \%$ & 4 & $7 \%$ & 6 & $6 \%$ & 0,765 \\
\hline Total & 46 & $100 \%$ & 54 & $100 \%$ & 100 & $100 \%$ & - \\
\hline \multicolumn{8}{|c|}{ Classe Social (Critério Brasil) } \\
\hline Classe D-E & 9 & $20 \%$ & 12 & $22 \%$ & 21 & $21 \%$ & 0,745 \\
\hline Classe C1 & 7 & $15 \%$ & 10 & $18 \%$ & 17 & $17 \%$ & 0,661 \\
\hline Classe C2 & 23 & $50 \%$ & 22 & $41 \%$ & 45 & $45 \%$ & 0,353 \\
\hline Classe B1 & 2 & $4 \%$ & 2 & $4 \%$ & 4 & $4 \%$ & 0,869 \\
\hline Classe B2 & 5 & $11 \%$ & 8 & $15 \%$ & 13 & $13 \%$ & 0,558 \\
\hline Classe A & 0 & $0 \%$ & 0 & $0 \%$ & 0 & $0 \%$ & $* * *$ \\
\hline Total & 46 & $100 \%$ & 54 & $100 \%$ & 100 & $100 \%$ & - \\
\hline
\end{tabular}

Siglas: IMC - índice de massa corporal. O símbolo (***) indica ausência de frequência com pontuação para classificação.

Persp. Online: biol \& saúde, Campos dos Goytacazes, 40 (11) 39-53, 2021

ojs3.perspectivasonline.com.br 
A Tabela 2 apresenta ainda os resultados da estatura para idade, nesta análise a grande maioria em ambos os grupos mostraram que a estatura está adequada para idade. Em adição, também é mostrado na referida tabela os resultados da avaliação de classe social na qual a maioria de ambos os grupos se apresentam na classe $\mathrm{C} 2$.

Além disso, foi realizada uma comparação entre os grupos de escolares masculino e feminino para os testes e quociente motor do protocolo KTK. Nesta análise mostrada na Figura 1 , os testes para força e agilidade, além do quociente apresentaram diferenças $(p<0,05)$ favoráveis ao sexo masculino, o que não ocorreu para o equilíbrio e noção espaço-temporal.

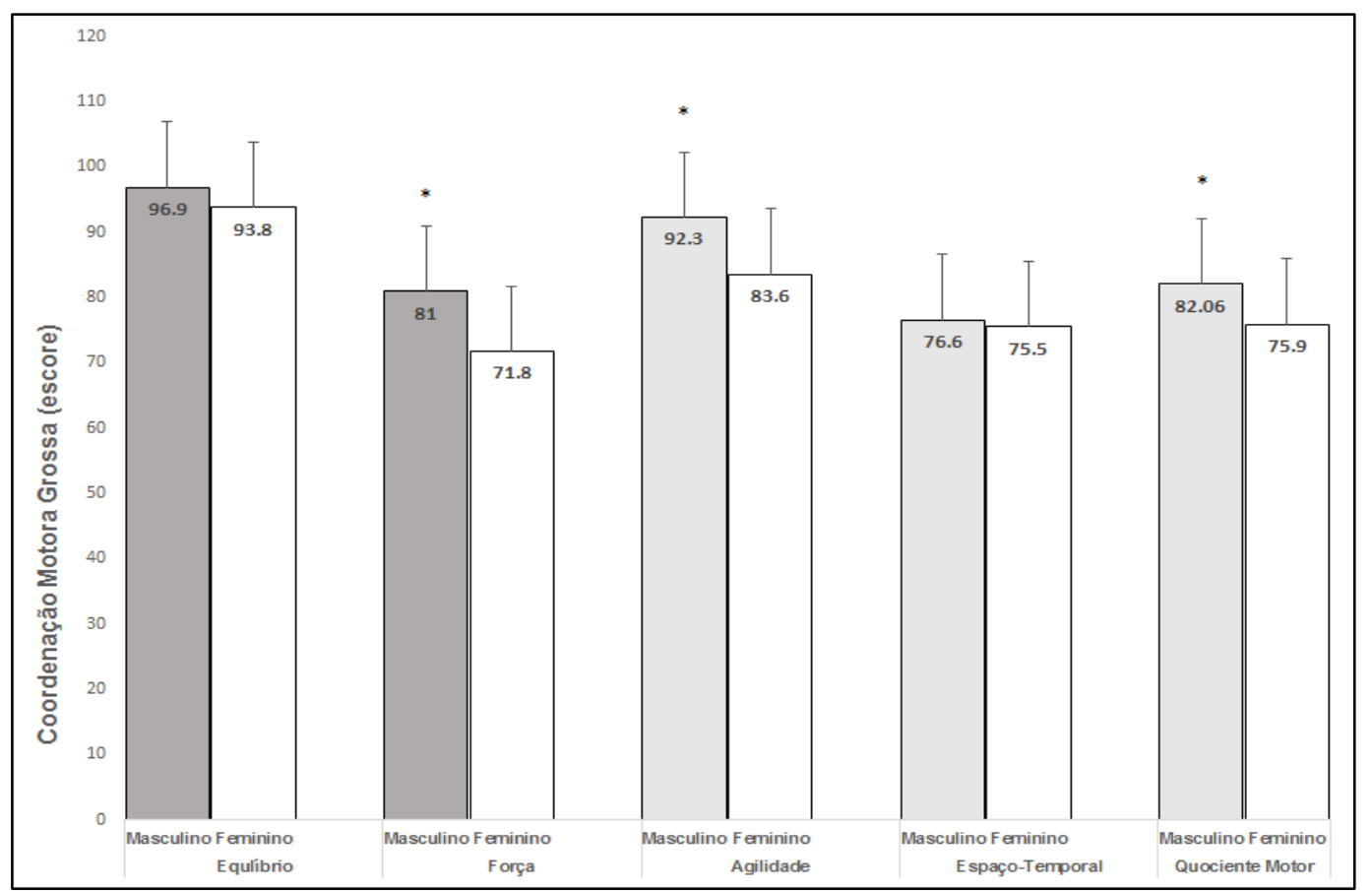

Figura 1. Resultados para os testes e quociente do protocolo KTK. O símbolo asterisco (*) indica um valor de $\mathrm{p}<0,05$.

\section{DISCUSSÃO}

O presente estudo, buscou descrever o perfil de coordenação motora grossa e estado nutricional de escolares com baixo nível socioeconômico de uma cidade da Amazônia brasileira, além de comparar as variáveis entre os sexos biológicos.

Ao avaliar a classificação geral da coordenação motora dos alunos, de ambos os sexos verificou-se que (24\%) se encontrava na classificação normal, (24\%) insuficiência e (52\%) Perturbação (Tabela 2). Além disso, nenhum aluno alcançou boa ou muito boa coordenação. No estudo de Santos (2016) que avaliou a coordenação motora de 108 crianças de ambos os sexos de 5 a 10 anos, foi verificado que a maioria (42,7\%) apresentou uma perturbação na coordenação, 38,2\% coordenação normal, 17,3\% insuficiência da coordenação e apenas 1,8\% boa coordenação, não havendo nenhuma criança com uma coordenação muito boa. Resultado semelhante também foi encontrado por Araujo (2015) que realizou um estudo com 82 crianças de 6 a 9 anos e verificou que a classificação obtida pelo teste KTK revelou que 15,9\% apresentaram insuficiência na coordenação, 41,5\% uma perturbação na coordenação, 40,2\% uma coordenação normal e $2,4 \%$ boa coordenação. Ambos os resultados dos estudos citados a

Persp. Online: biol \& saúde, Campos dos Goytacazes, 40 (11) 39-53, 2021 
cima são semelhantes aos apresentados na presente pesquisa, mostrando que a maioria dos escolares de ambos os sexos foram classificados em perturbação e insuficiência de coordenação.

O que vai de encontro ao apresentado por Souza et al. (2015) que avaliou dois grupos de crianças de 6 a 10 anos de bairros com níveis socioeconômicos distintos em uma cidade na Amazônia, mostrando nos resultados que a prevalência na classificação geral do KTK foi normal. Porém, os alunos de melhores condições socioeconômicas mostraram melhor coordenação motora $(\mathrm{p}<0,05)$ comparado ao grupo de menor condição, assim como o grupo apresentado na presente pesquisa que possui condições socioeconômicas baixas e coordenação motora com perturbação em sua maioria.

Entretanto, diferentes resultados foram encontrados por Santos (2014), que avaliou 91 crianças com idades entre 7 e 8 anos, observando que a maioria apresentou uma Boa coordenação motora $(46,15 \%)$, seguido de coordenação Normal $(45,05 \%)$ e $8,8 \%$ de crianças com Muito Boa coordenação, resultados melhores que os apresentados nesta pesquisa, e pode ser justificado pela realidade social dos países onde os estudos foram realizados, Portugal por exemplo, é um país de primeiro mundo, altamente desenvolvido e com Índice de Desenvolvimento Humano (IDH) muito à frente do Brasil que ocupa atualmente a posição 79 do ranking global da Organização das Nações Unidas (ONU), sendo um país considerado emergente e de segundo mundo, que ainda apresenta sérios problemas sociais.

Em relação aos testes de coordenação motora grossa e a diferença de sexos, a força e a agilidade, além do quociente motor apresentaram diferenças $(\mathrm{p}<0,05)$ favoráveis ao sexo masculino, o que não ocorreu para o equilíbrio e noção espaço-temporal (Figura 1). O estudo de Souza et al. (2015) corrobora com os resultados observados neste estudo, onde demonstrou que quociente motor geral do sexo masculino foi superior ao feminino.

Santos (2014) e Santos (2016) e revelaram também em seus estudos que o sexo masculino teve níveis mais elevados de coordenação em relação ao sexo feminino. Para Valdivia et al. (2008), essa predominância do sexo masculino se sobressair ao feminino nos níveis de coordenação motora, se justifica,-pelo fato do público feminino comumente estar envolvido em atividades menos ativas e destinarem menos tempo a estas práticas em relação ao público masculino. Os filhos homens têm mais liberdade dos pais, isso pode resultar em maiores possibilidades de momentos ativos na vida diária (VALDIVIA et al., 2008). Além disso, crianças do sexo masculino, mesmo entre oito e dez anos, podem encontrar-se em diferentes estágios de maturação sexual, e isso pode aumentar os níveis de hormônios sexuais como a testosterona (ARAÚJO; MEDEIROS, 2017), o que pode justificar a diferença $(p<0,05)$ nos testes de força e agilidade da presente pesquisa.

Em relação ao estado nutricional, verificou-se as variáveis estatura para idade e IMC para idade de acordo com OMS (YAN-PING et al 2009). Ao avaliar o estado nutricional dos participantes da amostra para variável IMC para idade, constatou-se que a maioria foi classificada em eutrófico (73\%), sobrepeso (14\%), obesidade (3\%) e magreza (3\%). Somente o sexo masculino apresentou resultados para obesidade (6\%) e magreza acentuada (1\%) (Tabela 1). No estudo de Chaves (2019) que analisou 272 crianças com idade entre 5 a 10 anos de ambos os sexos na Ilha de Cotijuba - PA, também apresentou a maior porcentagem (82\%) para classificação eutrófico.

Persp. Online: biol \& saúde, Campos dos Goytacazes, 40 (11) 39-53, 2021

ojs3.perspectivasonline.com.br 
Ainda relacionado ao IMC para idade, Sousa et al. (2014) analisando crianças de 7 a 11 anos, constataram que $10,29 \%$ estavam desnutridas, $3,4 \%$ acima do peso e $89,7 \%$ com peso normal. Entretanto, o estudo realizado no âmbito de estado nutricional de crianças demonstraram resultados que não contemplam os achados neste estudo. O estudo de Lima (2014) revelou números inferiores ao presente estudo, evidenciando em sua análise a classificação mais baixa na variável do estado nutricional para crianças ribeirinhas moradores de São Luiz do Tapajós, apontando maior frequência de baixo peso e baixa estatura. A razão disso, pode estar relacionada a infraestrutura e saneamento básico dessas localidades interioranas que são desprezadas pelos governantes.

Outra variável do estado nutricional avaliada foi a estatura para idade, onde constatouse que, a maioria foi classificada como adequada (94\%), baixa $(6 \%)$, sendo que não foi encontrada nos avaliados a classificação muito baixa (Tabela 1). É importante ressaltar que as crianças analisadas neste estudo receberam uma refeição por dia no horário de intervalo de aula, o que pode influenciar os resultados de estado nutricional satisfatórios como: Estatura adequada para idade e nível eutrófico na variável IMC para idade, mesmo considerando que os alunos possuem baixo nível socioeconômico. Resultado semelhante foi encontrado por Chaves (2019), onde, de sua amostra total $(95,6 \%)$ apresentaram-se com estatura adequada para a idade.

No perfil socioeconômico dos alunos, constatou-se que (45\%) encontra-se na Classe C2, (21\%) D-E, (17\%) C1, (13\%) B2, (4\%) B1. Nenhum dos investigados enquadrou-se na Classe A (Tabela 1). Semelhante a este estudo, Chaves (2019) destaca que a maioria das famílias das crianças estudadas em sua pesquisa apresentaram classe econômica C2 (32,7\%) e D (28,3\%). Ratificando assim, a vulnerabilidade socioeconômica desta população. Já, diferentemente deste estudo, Estevão e Da luz (2015) que avaliaram 216 meninos e meninas, verificaram que as classes predominantes foram B2 e C1. Apesar de ambos os estudos acontecerem em escolas da rede pública, essa disparidade nos resultados pode ser explicada pela diferença regional entre as pesquisas.

Souza et al. (2015) em seu estudo apresentaram para o nível socioeconômico que $38 \%$ dos meninos do "Grupo Vila" fazem parte das classes B1 e B2, já 43\% das meninas deste mesmo grupo, classificadas na classe B1. Enquanto para o "Grupo Cidade" tanto os meninos $(53 \%)$ quanto às meninas $(44 \%)$ foram classificados na classe D. Levando em consideração o resultado do "Grupo Cidade" este que se assemelha com público da presente pesquisa, observando-o que as crianças com menor nível socioeconômico apresentaram os piores resultados para coordenação motora grossa e estado nutricional.

Diante disto, observa-se que o baixo nível socioeconômico pode ser um fator potencial de influência em resultados negativos para coordenação motora grossa e estado nutricional de crianças em idade escolar. De acordo com os dados apresentados na pesquisa, observou-se um melhor desempenho do sexo masculino quanto a coordenação motora grossa, mas ainda longe do ideal, que pelos resultados observados podem não está associado ao estado nutricional, pois a maioria apresenta resultados positivos nas duas variáveis, porém o baixo nível socioeconômico que os mesmos encontram-se, talvez seja um fator relevante ao baixo nível de coordenação motora grossa em alguns aspectos conforme também foi evidenciado por Souza et al. (2015) e Zanato et al. (2018).

Os resultados observados neste estudo com relação à coordenação motora grossa, indicaram um déficit nesta variável, onde (76\%) da amostra apresentou algum tipo de perturbação ou insuficiência, que pode ter relação com o Transtorno de Desempenho da

Persp. Online: biol \& saúde, Campos dos Goytacazes, 40 (11) 39-53, 2021

ojs3.perspectivasonline.com.br 
Coordenação (TDC), pois estimativas internacionais apontam para uma prevalência deste problema em torno de $6 \%$ das crianças em idade escolar, com destaque para o sexo masculino (EDITION et al., 2013). Nos estudos brasileiros, não se encontrou um consenso da estimativa do TDC, com as prevalências variando em torno de 4,4\% a 19,9\% (SANTOS; VIEIRA, 2013; VALENTINI et al., 2012). De acordo com a OMS o TDC é denominado de transtorno específico do desenvolvimento da função motora, caracterizado como um forte atraso no desenvolvimento da coordenação motora, com desempenho consideravelmente abaixo do adequado para a idade cronológica e nível cognitivo da criança, nas atividades diárias que envolvem essa variável (EDITION et al., 2013).

Estudos vêm investigando a prevalência do TDC em crianças com idade escolar no Brasil. Silva e Beltrame (2013) constataram a prevalência de $11 \%$ de problemas motores em 406 crianças entre 7 e 10 anos de uma escola municipal de São José/SC. Valentini et al. (2012) realizou outro estudo no (Rio Grande do Sul, Paraná e Santa Catarina) com 1.587 crianças entre 4 e 12 anos de idade, que ao final chegou ao resultado que $19,9 \%$ das crianças são prováveis TDC e outros 16,8\% tem risco de serem acometidos pelo transtorno. Melo et al. (2014) realizou um estudo no município de Ceilândia-DF, e observou que $8 \%$ da população estudada tinha TDC.

No estudo desenvolvido por Franca et al. (2017) que avaliou 535 crianças de João Pessoa-PB, constatou que 253 obtiveram pontuação indicando problemas motores, possível TDC, 200 delas (79\%) se encontram nas classes econômicas C, D, e E, podendo ser consideradas como famílias com baixo poder aquisitivo. A relação relevante de provável TDC com baixo nível socioeconômico sugere que o ambiente pode ter influência no desenvolvimento motor, uma vez que crianças de famílias com baixo poder aquisitivo podem receber menos estímulos, situação semelhante observada neste estudo. Embora o TDC não tenha sido avaliado nesta pesquisa, vale destacar que a maior parte dos alunos apresentaram déficit de coordenação verificado nos resultados e isso pode ser um indicativo para maiores cuidados com as crianças estudas, dado aos estudos citados a cima que mostram uma possível associação do TDC com o baixo nível socioeconômico e com o déficit de coordenação motora.

Contudo, o combate aos múltiplos problemas de saúde existentes na infância que associam as variáveis de estado nutricional e a coordenação motora grossa deveriam ser prioridades em estratégias de políticas públicas para esta faixa de idade, em especial, para comunidades de menor nível socioeconômico (EDITION et al., 2013; ZANATO et al., 2018; VANDENDRIESSCHE et al., 2012), sinalizando para um perigo eminente de múltiplos problemas gerados por esta falta de cuidados. Diante disto, sugere-se novos estudos que comtemplem um maior número amostral de crianças, que também possa realizar uma análise de correlação estatísticas entre estas variáveis para verificação de possívis associações estatísticas entre as mesmas.

\section{CONCLUSÕES}

O estudo mostrou que crianças de 8-10 anos de uma escola pública em um bairro na periferia de Tucuruí-PA, encontram-se com a coordenação motora grossa abaixo da normalidade conforme o protocolo KTK, mesmo o sexo masculino tendo obtido melhores resultados para força e agilidade. No que tange o estado nutricional, os mesmos apresentaram resultados satisfatórios de acordo com a proposta da OMS nas variáveis (IMC/Idade) e (Estatura/Idade), embora o nível socioeconômico aponte vulnerabilidade do público em

Persp. Online: biol \& saúde, Campos dos Goytacazes, 40 (11) 39-53, 2021

ojs3.perspectivasonline.com.br 
questão. Neste caso, infere-se que o nível socioeconômico pode ser uma causa do baixo nível de coordenação motora grossa de escolares de 8-10 anos.

\section{REFERÊNCIAS}

ARAÚJO, F. S. D. Avaliação da coordenação motora em crianças dos 6 aos 9 anos de idade. 2014. 72 f. Dissertação (Mestrado) - Curso de Educação Física, Ciências de Desporto, Exercício e Saúde, Universidade de Trás os Montes e Alto Douro, Vila Real, 2015. Disponível em: https://repositorio.utad.pt/handle/10348/5136

ARAÚJO, J. P. F.; MEDEIROS, J. A. Comportamento da coordenação motora de escolares em diferentes estágios maturacionais. Revista UNI-RN, Natal, v.16 v.17, suplemento, p. 113-137, jan./dez. 2017. Disponivel em: https://eventos.set.edu.br/CIAFIS/article/view/2627

ASSOCIAÇÃO BRASILEIRA DE EMPRESAS DE PESQUISA - ABEP. Critério de classificação econômica Brasil. 2015. Disponível em: https://www.abep.org

BRANCO, L.; GUILHERM, G.; CALOMENI, M. R. O efeito de estratégias pautadas nos neurônios espelho e atividades rítmicas no desenvolvimento psicomotor de crianças em processo de alfabetização. Biológicas \& Saúde, v. 8, n. 27, 14 nov. 2018. Disponível em: https://ojs3.perspectivasonline.com.br/biologicas_e_saude/article/view/1472

BRASIL. Conselho Nacional de Saúde. Diretrizes e Normas Regulamentadoras de Pesquisas Envolvendo Seres Humanos. Resolução 466/12, 2012. Disponível em: https://conselho.saude.gov.br/resolucoes/2012/Reso466.pdf

CONSTANTINO-COLEDAM, D. H. et al. Associação entre indicadores socioeconômicos com a atividade física e aptidão física relacionada à saúde em adolescentes. Revista de Salud Pública, Bogotá, v. 15, n. 6, p. 823-836, 2013. Disponível em: https://www.scielosp.org/article/rsap/2013.v15n6/810-815/

GALLAHUE, David L.; OZMUN, John C.; GOODWAY, Jackie D. Compreendendo o desenvolvimento motor-: bebês, crianças, adolescentes e adultos. AMGH Editora, 2013. Disponível em: https://books.google.com.br/books?hl=pt$\mathrm{BR} \& \mathrm{lr}=\& \mathrm{id}=\mathrm{R} 6 \mathrm{xIAgAAQBAJ} \& \mathrm{oi}=\mathrm{fnd} \& \mathrm{pg}=\mathrm{PR} 3 \& \mathrm{dq}=$ Compreendendo+o+desenvolvimento +motor:+beb\%C3\%AAs,+crian\%C3\%A7as,+adolescentes+e+adultos\&ots=b1XKjXNUU6\&s ig=HE4e-

7EUX9_K0ujRS5F5jBjdaYA\#v=onepage \&q=Compreendendo $\% 20 \mathrm{o} \% 20$ desenvolvimento $\% 2$ 0motor\%3A\%20beb\%C3\%AAs \%2C\%20crian\%C3\%A7as\%2C\%20adolescentes\%20e\%20ad ultos $\& \mathrm{f}=$ false

EDITION, Fifth et al. Diagnostic and statistical manual of mental disorders. Am Psychiatric Assoc, v. 21, 2013. Disponível em: http://repository.poltekkeskaltim.ac.id/657/1/Diagnostic\%20and\%20statistical\%20manual\%20of\%20mental\%20disorde rs\%20_\%20DSM-5\%20\%28\%20PDFDrive.com\%20\%29.pdf Acesso em 18 abril 2021

Persp. Online: biol \& saúde, Campos dos Goytacazes, 40 (11) 39-53, 2021

ojs3.perspectivasonline.com.br 
ESTEVÃO, B.J.; DA LUZ, R. F. Coordenação motora, nível socioeconômico e a prática esportiva extraclasse: um estudo da rede pública de ensino. Educação Física em Revista, v. 8, n. 2, 2015. Disponível em: https://portalrevistas.ucb.br/index.php/efr/article/view/3272/3953

FRANCA, A. S.; CARDOSO, A. A.; ARAÚJO, C. R. S. Problemas de coordenação motora e de atenção em crianças em idade escolar. Revista de Terapia Ocupacional da Universidade de São Paulo, v. 28, n. 1, p. 86-92, 2017. Disponível em: https://www.revistas.usp.br/rto/article/view/121303/129283

GIORDANI, L. G.; ALMEIDA, C. S.; PACHECO, A. M. Avaliação das oportunidades de desenvolvimento motor na habitação familiar de crianças entre 18 e 42 meses. Motricidade, v.9 n.3, p. 2013. 96-104, Disponível em: https://lume.ufrgs.br/bitstream/handle/10183/94569/000912018.pdf?sequence=1\&isAllowed= $\mathrm{y}$

GORE, F. M. et al. Global burden of disease in Young people aged 10-24 years: a systematic analysis. Lancet, London, v. 377, no. 9783, p. 2093-2102, 2012. Disponível em: https://www.thelancet.com/journals/lancet/article/PIIS0140-6736(11)60512-6/fulltext

GORLA, J. I.; ARAUJO, P. F.; RODRIGUES, J. L. O teste KTK em estudos da coordenação motora. Conexões: Revista da Faculdade de Educação física da UNICAMP, vol. 01, n. 01, $2003 . \quad$ Dispinível em: https://periodicos.sbu.unicamp.br/ojs/index.php/conexoes/article/view/8640804

GUSMÃO, S. Redação de artigo científico. Arquivos Brasileiros de Neurocirurgia, São Paulo, v. 30, n. 2, p. 44-50, 2011. Disponível em: https://publicacoes.unifunec.edu.br/wpcontent/uploads/2017/07/redac\%CC\%A7a\%CC\%83o-de-um-artigo.pdf

IBGE. Instituto Brasileiro de Geografia e Estatística. Coordenação das Estatísticas Econômicas e Classificações por Grandes Categorias Econômicas CGCE - IBGE. Rio de Janeiro: IBGE, 2013. Disponível em: https://www.ibge.gov.br/estatisticas/metodos-eclassificacoes/classificacoes-e-listas-estatisticas/9147-classificacao-por-grandes-categoriaseconomicas.html

LIMA, A. C. M. Estado nutricional e desenvolvimento motor de crianças ribeirinhas expostas ao mercúrio no estado do Pará- Amazônia Brasileira. 2014. 72 f. Tese (Doutorado) - Núcleo de Medicina Tropical, Programa de Pós-Graduação em Doenças Tropicais, Universidade Federal do Pará, Belém, 2014. Disponível em: http://repositorio.ufpa.br/jspui/handle/2011/9115

CHAVES, L.S.; Estudo do perfil socioeconômico, estado nutricional, nível de mercúrio e desenvolvimento motor em crianças da Ilha de Cotijuba, Pará / Brasil . Belém-Pará: ed 1: Conhecimento \& Ciência, 2019. Disponível em: https://drive.google.com/file/d/16u1UzbNDmDlieotliSv7oy5jokXzOMf/view

MELO, T. F. M. et al. Influência da educação integral na prevalência de transtorno do desenvolvimento da coordenação em crianças em idade escolar. Cadernos de Terapia Ocupacional da Ufscar, [s.1.], v. 22, n. 3, p.537-542, 2014. Editora Cubo Multimidia.

Persp. Online: biol \& saúde, Campos dos Goytacazes, 40 (11) 39-53, 2021

ojs3.perspectivasonline.com.br 
Dsiponível

http://www.cadernosdeterapiaocupacional.ufscar.br/index.php/cadernos/article/view/798

RIBEIRO, A. S. et al. Teste de coordenação corporal para crianças (KTK): aplicações e estudos normativos. Motricidade, Santa Maria da Feira, v. 8, n. 3, p. 40-51, 2012. Disponível em: https://www.redalyc.org/pdf/2730/273024354010.pdf

SANTOS, C. N. D. Fatores que influenciam a coordenação motora em crianças dos 5 aos 10 anos. 2016. Dissertação (Mestrado em Educação Física e Desporto) - Departamento de Ciências de Desporto, Exercício e Saúde, Universidade de Trás os Montes e Alto Douro, Portugal. Disponível em: https://repositorio.utad.pt/handle/10348/6255

SANTOS, J. R. C. D. A influência da classe socioeconômica e obesidade na coordenação motora em alunos de 7 e 8 anos de idade. 2014. Dissertação (Mestrado em Educação Física e Desporto) - Departamento de Ciências de Desporto, Exercício e Saúde, Universidade de Trás os Montes e Alto Douro, Portugal. Dsiponível em: https://ubibliorum.ubi.pt/bitstream/10400.6/5858/1/4568_8632.pdf

SANTOS, V. A. P.; VIEIRA, J. L. L. Prevalência de desordem coordenativa desenvolvimental em crianças com 7 a 10 anos de idade. Revista Brasileira de Cineantropometria e Desempenho Humano, Florianópolis, v. 15, n. 2, p. 233-242, 2013. Disponível em: https://www.scielo.br/j/rbcdh/a/39SxWPKRFF9vXzKZtmBVTZn/?format=pdf\&lang=pt

SILVA, J.; BELTRAME, T. S. Indicativo de transtorno do desenvolvimento da coordenação de escolares com idade entre 7 e 10 anos. Revista Brasileira de Ciências do Esporte, [s.1.], v. 35, n. $1, \quad$ p.3-14, 2013.2 Disponível https://www.scielo.br/j/rbce/a/hKzdsCTGfGnPqcDGbfYdW7G/abstract/?lang=pt

SOUSA, J. M. et al. Assessment of the motor development of children poisoned with mercury. Gazzetta Medica Italiana Archivio per le Scienze Mediche, v. 173, p. 1-2, 2014. Disponível em:

https://d1wqtxts1xzle7.cloudfront.net/36493369/Josenaldo_tese_Assessment_of_the_motor_d evelopment-with-cover-page-

v2.pdf?Expires $=1635258268 \&$ Signature $=$ ZRzU82idcoz0KmYu6VgbMT8-

9yIJaoXmPTC6ZVL2kgDNcTQCemSTCkLQqG4fo ARQ8ISXOrVZWnIjNqbSavWqt8TV4 cOYs0miO9F-vqHtYTu0VuocQDaPFkfolDiC4fn-

kEnSLmwV8pfzMr Fj8QK0Qd0sXQJ51xL9GzwP8jauLb21Jg1RS924uBbxVkfbA0KFI39Eei kubrakNUCwOTbKLWsVU3DZBP51JABY9a82bJ0v3nmuO-

8vtZZsfYz8hpksCZers8gvLMIBo6wa3MjVksRZe5s4Wj6z58ISUVOx8Xn7jBVNz2qMe0gL HQxwa076pa-E2OBhcuV4cH8LA4ig_\&Key-Pair-Id=APKAJLOHF5GGSLRBV4ZA

SOUZA, N. S. N., et al. Nível socioeconômico, estado nutricional e coordenação motora grossa de escolares com 6 a 10 anos na Amazônia. Revista de Educação Física/UEM, v. 26, n. 3, p. 401-411, 3. Trim, 2015. Disponível em: https://www.scielo.br/j/refuem/a/KxcJDvVV7RPfSDpLrzXSnGm/?format=html\&lang=pt

YAN-PING, Li et al. Application of the WHO growth reference (2007) to assess the nutritional status of children in China. Biomedical and Environmental Sciences, v. 22, n. 2, p. 130-135, 2009. Disponível em: https://www.sciencedirect.com/science/article/pii/S0895398809600350

Persp. Online: biol \& saúde, Campos dos Goytacazes, 40 (11) 39-53, 2021

ojs3.perspectivasonline.com.br 
VALDIVIA, A. B., et al. Coordinación motora: influencia de la edad, sexo, estatus socioeconómico y niveles de adiposidad en niños peruanos. Revista Brasileira de Cineantropometria Desempenho Humano, v. 10, n. 1, p. 25-34, 2008. Disponível em: https://www.researchgate.net/profile/Andre-

Seabra/publication/26497500_Motor_coordination_Influence_of_age_sex_socioeconomic_status_and_levels_of_adiposity_in_peruvian_children/links/00b4951f663664ee0d0 00000/Motor-coordination-Influence-of-age-sex-socio-economic-status-and-levels-ofadiposity-in-peruvian-children.pdf

VALENTINI, N. C. et al. Prevalência de déficits motores e desordem coordenativa desenvolvimental em crianças da região Sul do Brasil. Revista Paulista de Pediatria, São Paulo, v. 30, n. 3, p. 377-384, 2012. Disponível em: https://www.scielo.br/j/rpp/a/yPbfF74JnJhZPn6mkzZTrKC/?lang=pt\&format=pdf

VANDENDRIESSCHE, J. B. et al. Variation in sport participation, fitness and motor coordination with socioeconomic status among Flemish children. Pediatric Exercise Science. Ontario, v. 24, no. 1, p. 113-128, 2012. Disponível em: https://biblio.ugent.be/publication/2320175/file/6773598.pdf

ZANATO, L.N; DE OLIVEIRA, K.R; CALÁBRIA, L. K. Perfil antropométrico, estado nutricional e nível sérico de ferro em assentados de Ituiutaba-MG. Biológicas \& Saúde, v. 8, n. 27, 32 out. 2018.2 Disponível https://ojs3.perspectivasonline.com.br/biologicas_e_saude/article/view/1379 